\title{
Regulation of Mirna Pathway and Roles of Micrornas in Tumorigenesis and Metastasis
}

\author{
Rong Jiang and Yujing Li* \\ Department of Human Genetics, Emory University School of Medicine, 615 Michael St, Atlanta, GA 30322, USA
}

\begin{abstract}
Although small non-coding RNAs, particularly microRNAs (miRNAs), small interference RNAs (siRNAs), and piwiinteracting RNAs (piRNAs), account for only a minor fraction of the expressed genome, they have been recognized as important regulators of gene and genome at post-transcriptional levels, functioning as RNA interference (RNAi) to regulate some of the most important biological processes in eukaryotic cells. Following the identification of the major components within the RNAi/miRNA pathway, some protein co-factors have been characterized to play significant roles in activity regulation of the RNAi/miRNA pathway in the last few years, suggesting that any regulators must be tightly regulated. It has been shown that microRNAs play vital roles in regulation of multiple signaling pathways and are involved in a variety of physiological and pathological processes. Given that high percentage of the identified human miRNAs-coding genes are located at tumor-related fragile chromosome regions, and that an increasing body of evidence supports the strong link between aberration of miRNA expression and malignancies, the regulatory network organizing the miRNAs, miRNA regulating factors and miRNA targets particularly the tumor suppressors or oncogenes is suggested. This review highlights the current evidence for the significance of the link of miRNAs and tumorigenesis and metastasis.
\end{abstract}

Keywords: microRNA; Regulation; Tumorigenesis; Metastasis; Mechanism

\section{Introduction}

Small non-coding RNAs, identified so far including small interference RNA (siRNAs), microRNAs (miRNAs), piwi-interacting RNA (piRNAs), promoter-associate (paRNAs), transcription initiation tiRNA, and tRNA-derived RNA fragments (tRFs), are 18-30 nucleotides in length. Although they account for only a very small fraction of the expressed genome, these small RNAs, have been acknowledged as pivotal regulators at post-transcriptional levels to control diverse biological pathways relating to chromosome architecture and segregation behavior, transcription, and RNA processing and stability, and thereby to phenotypically coordinate development, growth control, apoptosis, self-defense, and stem cell maintenance [1-6]. Accordingly, as expected, increasingly obvious evidences demonstrate that alteration of miRNAs expression profile could contribute to the pathogenesis of a wide range of human diseases as well as phenotypic abnormality among the eukaryotes [7]. Moreover, siRNA/miRNA based knockdown has been technically adopted in a wide range as powerful tools to elucidate gene functions, to discover and validate the drug targets, and to develop therapeutic intervention. Given the pivotal roles of siRNA/miRNA in diverse biological pathways as well as the broad application of RNAi, it is essential to understand the siRNA/miRNA pathway and its regulation mechanism. Since the identification of the major components of the RNAi/miRNA pathway [2], focus has been switched to the regulation mechanism of the pathway itself in recent years, resulting in discovery and characterization of co-factors functioning as regulators of the RNAi/miRNA pathway at different levels from pre-transcription to post-transcription.

\section{miRNA Processing Pathway}

In mammals, majority of miRNA genes are transcribed initially by RNA polymerase II as primary transcripts (pri-miRNAs) range from hundreds to thousands of nucleotides in length that bear one or more hairpin structures [8]. The pri-miRNAs are 5'-capped, spliced and polyadenylated $[5,9,10]$. Subsequently, two sequential biochemical processing steps lead to generation of mature form of miRNAs. In most cases, the first step takes place inside the nucleus where the primiRNAs were cropped by Drosha, a RNA endonuclease III in mammals and its partner DGCR8 [10] to produce a $70 \mathrm{nt}$ stemloop-structured precursor (pre-miRNAs) that harbors the miRNA in the 5' or 3' half of the stem $[8,11]$. However, this is not the only way of pre-miRNAs biogenesis. Alternatively, generation of some pre-miRNAs can bypass the Drosha processing step. One example is "mirtrons," the premiRNA-like hairpins which are generated by splicing and debranching of the short hairpin introns [12-14]. Besides "mirtron", some small nucleolar RNAs (snoRNAs), tRNAs, and endogenous short hairpin RNAs (shRNAs) are processed into miRNA-like molecules without relying on Drosha processing either [15-18]. More recently another source of miRNAs, terminal hairpins of endogenous siRNA long-stemloop precursors, has been identified as being able to bypass the Drosha processing step [19].

The pre-miRNAs are transported into cytoplasm by exportin-5/ RanGTP for the secondary processing [8,10,20,21]. The second processing step occurs in the cytoplasm where the pre-miRNAs are further diced into mature form of miRNAs with length of 18-25 nt by Dicer, another member of RNase III family in collaboration with its cofactors TRBP and PACT [8,22,23].

Once maturated, the guide/functional strand and the passenger strand of the miRNA duplex are facing different fates. It is generally

*Corresponding author: Yujing Li, Department of Human Genetics, Emory University School of Medicine, 615 Michael St, Atlanta, GA 30322, USA, E-mail: yli29@emory.edu

Received January 23, 2013; Accepted February 04, 2013; Published February 06,2013

Citation: Jiang R, Li Y (2013) Regulation of Mirna Pathway and Roles of Micrornas in Tumorigenesis and Metastasis. Human Genet Embryol S2: 007. doi:10.4172/2161-0436.S2-007

Copyright: ( 2013 Jiang R, et al. This is an open-access article distributed under the terms of the Creative Commons Attribution License, which permits unrestricted use, distribution, and reproduction in any medium, provided the original author and source are credited. 
believed that the guide strand is sorted to load into RISC complex to regulate gene expression at post-transcriptional levels, while the passenger strand is finally degraded [24]. However, recent deep sequencing results in Drosophila have indicated that a large number of passenger strands are functional and associated with AGO1 or AGO2 instead of being degraded [25-28]. Of the sequence features determining the selection of guide strands, the thermodynamic asymmetry of the miRNA duplex contributes greatly to the sorting process [29,30], while other features such as the central mismatches and the identity of the 5 ' nucleotide affect sorting as well. The mature form of miRNAs function as recognizer once they are loaded into the RNA induced silencing complex (RISC) to match their target mRNAs through base-pairing. The RISC consists of several components with AGO protein as core for RNA silencing $[5,31,32]$.

\section{Regulation of microRNA Pathway}

Any essential regulators must be tightly regulated to maintain normal cellular homeostasis. Similarly, misregulation of miRNAs/ siRNAs expression levels alters their target levels, severely affecting normal cellular metabolism. To precisely regulate the expression levels of individual miRNA/siRNA, a serial of regulatory mechanisms along the miRNA/siRNA pathway have been discovered at levels of genome, epigenome, and transcriptome.

\section{Regulation at Level of miRNA Gene Sequences}

Several events at the DNA level contribute to alteration of miRNA biogenesis such as copy number change or mutations in the miRNA genes. So far very few reports are available at this level and most of them are found in human cancer cells [33]. Mutations were identified in 5 of 42 tested miRNA genes in patients with chronic lymphocytic leukemia (CLL), but not in the normal healthy controls. More specifically, mutation of 7 bp downstream from pre-miR15a 16-1 genomic DNA in germline, inhibited processing. A more common form of point mutations is the single nucleotide polymorphism (SNP) which alters the miRNA processing and in some cases the miRNA functions as well. It has been shown that 315 SNPs were associated with 265 human miRNA genes and only 12 of these SNPs were found in miRNA precursor sequences, and one SNP was located in the miR125a seed sequence [34]. Some SNPs could confer the differences in the Droshamediated processing such as miR125a precursor SNPs, miR-146a, miR502, miR-510, miR-890, and miR-892b [34-36], while differences were observed in the Dicer-conferred processing in some other SNPs such as miR-196a G/T SNPs as well [37]. However, mutations including SNPs are passive consequences of the mutations occurred in the miRNA genes. Therefore, it is not an active/auto miRNA processing regulation mechanism but rather pathologically associated with some human diseases. For example, miR-125a SNP is highly associated with breast cancer tumorigenesis [16]. Thus, SNPs could serve as pathological markers clinically.

\section{Regulation at Epigenetic Level}

Some epigenetic factors that affect transcription such as histone deacetylation and hypermethylation of promoter region of miRNA gene may also significantly inhibit or even completely silence the biogenesis of miRNAs. An example is that hypermethylation of miR-127 gene promoter region results in either dramatic inhibition or completely silencing of the miR-127 expression in many kinds of tumors more evidenced by the fact that treatments of these tumor cells with agents of demethylation and histone deacetylase inhibitor dramatically increase the miR-127 levels [38].

\section{Regulation at Transcriptional/Posttranscriptional Levels}

Regulations of miRNA processing at post-transcriptional level are thought to be the most important part of the regulation mechanisms. Several factors contribute to the regulations at RNA level. One of the factors is the modification of pri-/pre-miRNA sequences which affect miRNA processing but do not alter activity of the RNAi/ miRNA processing machinery. A more common mechanism is the alteration of the activity of the RNAi/miRNA processing machinery. Modulations of the RNAi/miRNA processing machinery activity are conferred by protein-protein interactions such as the co-factors that interact with Drosha and Dicer, or by protein-RNA interactions such as the co-factors that interact with the terminal loops of the pri- and/ or pre-miRNAs. Indeed, more and more protein components are being identified to enhance or inhibit the miRNA processing efficiency at post transcription level.

\section{Modifications of pri-/pre-miRNA sequences}

Pri-/pre-miRNA sequence modifications, typically RNA editing could alter the miRNA processing efficiency. The editing characterized so far is conversion of adenosine (A) to inosine (I) by adenosine deaminases on RNA (ADARs) in dsRNA structures [39,40]. Two isoforms of ADAR, ADAR1 and ADAR2 are responsible for the editing. The editing takes place in both pri-miRNAs and pre-miRNAs and most likely in nuclei due to the predominant nuclear localization of ADARs. In addition, the pre-miRNAs may also be edited in cytoplasm, caused by ADARs shuttle in and out of nucleus $[29,40]$. The edited pri-miRNAs may block the Drosha-mediated processing such as primiR-22 and primiR-142 because the editing occurred at the position surrounding the Drosha cleavage site $[41,42]$. However, some edited primiRNAs such as pri-miR-142 and pri-miR-151 do not affect the Drosha-mediated processing but rather hinder the Dicer-mediated processing [29]. The editing does not only affect the miRNA processing, but also interfere with the miRNA functions due to alterations of the "seed" region crucial for the recognition and binding of the RISC to targets. This interference has been confirmed in the edited miR-376 [29]. Even though the edited pri-miRNAs could not be appropriately processed by Drosha or Dicer, their accumulation was not detectable in nucleus, suggesting that specific degradation mechanism must be adopted to maintain homeostasis. Under in vitro conditions, the edited pri-miR142 could be cleaved by Tudor-SN [42]. It has been shown that Tudor-SN is a component of RISC and has ribonuclease activity specific to inosinecontaining dsRNAs [43]. However, so far it is still lacking evidence for in vivo relevance of the degradation of the edited pri-miRNA by TudorSN. Since the ADARs-based editing is not a pathological event at least based on the current research data, this represents an auto-regulation mechanism. However, the physiological significances are not clear and the fate of the edited miRNAs remains to be elusive.

\section{Co-factors interaction with major components of the RNAi/ miRNA pathway}

Of the co-factors regulating miRNA processing, the DEAD-box RNA helicase p68 and p72, central tumor suppressor p53, nuclear factors NF90 and NF45, Ars2 and CBC, and the splicing factors SF2/ ASF have been shown to modulate the Drosha-mediated primiRNA processing [44-50]. More specifically, the p68/p72 complex has dual functions: enhancement or inhibition in regulations of the Droshamediated processing of a subset of miRNAs depending on the protein components with which the p68 interacts $[45,48,51]$. Interaction of p68 with p53 or SMAD, induced by transforming growth factor $\beta$ (TGF- $\beta$ ) and bone morphogenetic proteins (BMPs), could facilitate the 
10 processing through promoting recruitment of Drosha complex to the target pri-miRNAs. On the other hand, interaction with estrogen receptor alpha $(E R \alpha)$ inhibits the pri-miRNA processing. In addition to p68/p72 complex, Ars2 and CBC are believed to serve as recruitment factors to enhance the pri-miRNA processing as well by binding the microprocessor [46]. More recently, it was found that the splicing factors SF2/ASF could directly interact with Drosha to facilitate Drosha-mediated processing of a subset of pri-miRNAs such as miR-7, miR-221, miR-222, and so on [52].

In addition to the positive regulators for the Drosha-mediated processing, a negative regulator complex NF90/NF45 was found to inhibit the processing step by reducing the accessibility of DroshaDGCR8 to pri-miRNAs rather than by interaction between NF90/ NF45 and the endogenous Drosha-DGCR8, evidenced by the high affinity of the NF90/NF45 to pri-miRNAs in Drosophila versus the lack of available data to show its interaction with Drosha-DGCR8 complex at present. Unexpectedly, while overexpression of NF90/ NF45 in 293 cells enhanced accumulation of pri-let-71-1, primiR- 21 and pri-miR-15a 16-1, respectively, the mature miRNA levels were not significantly altered [47], suggesting that the unknown mechanism(s) is in compensation with decreased pri-miRNA processing efficiency induced by NF90/NF45 overexpression. Given the dual regulations of the Drosha-mediated primiRNA processing by positive and negative regulators, it is rationale to believe that the balance between contributions made by the positive and negative co-factors involved in the processing could maintain the homeostasis of the pri-miRNAs, premiRNAs, and the mature miRNAs.

Like in the first step processing, more co-factors/signaling pathways are also involved in the Dicer-mediated pre-miRNA processing. TRBP and PACT were first characterized as cofactors not essential to the processing but facilitate the RISC assembly. However, further study showed that TRBP phosphorylation mediated by the mitogen activated protein kinase (MAPK) signaling pathway could stabilize the Dicer-containing complex [53]. This is the first report regarding posttranslational regulation of miRNA/RNAi pathway. Another co-factor, Wig-1, a p53-induced zinc finger protein can interact directly with Dicer to increase the activity of Dicer [54].

\section{Regulation by Argonaute protein family members}

Argonaute protein family members (AGO1-4), particularly AGO2 in mammals are confirmed to function as RISC-based mRNA cleavage or translational repression. Surprisingly, these AGO members themselves also enhance the miRNA processing, evidenced by the fact that ectopic overexpression of Ago1-4 significantly elevated levels of some mature miRNAs such as miR-215, miR-17-5p, miR-23b, and miR-92 [55]. What is more, using its intrinsic endonuclease activity in mammals, like Dicer, AGO2 could cleave the pre-miRNAs to an additional processing intermediate which may facilitate miRNA duplex dissociation and formation of RISC complex [55]. More recently, two groups found a Dicer-independent but Argonaute 2 catalytic activitydependent novel miRNA processing pathway [56,57]. In vivo and in vitro data indicated that pre-miR-451 was loaded into RISC complex and cleaved by $\mathrm{AGO} 2$ followed by uridylation and trimming to generate functional miRNAs. This novel pathway might not be a main pathway for miRNA biogenesis, but it links the conservation of Argonaute catalysis to a conserved mechanism of miRNA biogenesis [56,57].

\section{Co-factors interaction with RNA stem loop structure}

Some co-factors regulate miRNA processing not only through interaction with Drosha or Dicer but also through binding with terminal loop structures in the pri-miRNAs or premiRNAs. One RNA terminal loop binding protein involved in pri- and pre-miRNA processing is the KH-type splicing regulatory protein (KSRP) [58], a component of both Drosha and Dicer complexes. KSRP binds to the terminal loop of its target pri-and pre-miRNAs (eg. miR-21, miR-16, miR-155, and let-7a) and enhances Drosha and Dicer-mediated processing through interactions with Drosha and Dicer [58]. In contrast to these positive cofactors, Lin 28 , a developmentally regulated RNA binding protein was identified to selectively inhibit the processing of pri-let- 7 by competing for binding to the conserved nucleotides in the loop of pri-let-7 [59-62]. Another mechanism that Lin 28 negatively modulates processing of prelet-7 is adding an oligouridine tail to the pre-let-7 in cytoplasm [63]. It has been proven that Lin 28 itself does not uridylate pre-let- 7 but it could recruit terminal uridylyl transferase 4 (TUTase 4 , or TUT4), which has been demonstrated to be responsible for the uridylation [63-65]. Another RNA binding protein that so far has been proven to be involved in miRNA processing is heterogeneous nuclear ribonucleoprotein A1 (hnRNP A1). hnRNP A1 has been indicated to facilitate processing of pri-miR-18a, a member of miR17-92 cluster by binding to both the terminal loop and a region in the stem [66,67]. However, so far no report is available for the effect of hnRNP Al on processing of premiR-18a. In addition to pri-miRNA-18a, other primiRNAs such as pri-let-7a-1 and pri-miR-101-1 are also found to be the binding targets of hnRNP A1, but no evidences have shown the hnRNP A1-conferred enhancement or inhibition of the processing in let-7a-1 and miR-1011. Since only few RNA terminal loop binding proteins and few pri- and pre-miRNAs were tested for processing efficiency alterations, it is too early to draw a conclusion on whether the terminal-loop RNA binding protein-mediated modulation of the miRNA processing efficiency is a common regulation mechanism.

\section{Regulation of mature miRNA stability}

Accumulation of mature form of miRNAs depends on regulations at levels of transcription, processing, recycling and turning over. While it has been acknowledged that regulation at any of these processing steps is a key determinant, the stability of mature miRNAs after processing serves as an important element for the homeostasis of functional miRNAs. Several recent findings address the mechanisms for controlling the decay of mature miRNAs. The first factor that affects the stability of mature miRNAs is Argonaute protein levels [55,6870]. In addition to functioning as enhancement of biogenesis from pre-miRNAs to mature miRNAs in some cases, Argonaute proteins can protect miRNAs from degradation by taking guide strand as "refugees". The second factor affecting mature miRNAs is the protective modifications such as methylation [71], uridylation [72], adenylation [73-76], diverse nucleotide substitutions, additions, and deletions [73-75,77]. The methylation catalyzed by HEN1 methyltransferase to add methyl group to the 3'ends of miRNAs is the first characterized mechanism for mature miRNAs modification in Arabidopsis [71]. The HEN1 mutation conferred substantially decreased miRNA levels and the enhanced uridylation of the 3'ends, suggesting the methylation of the 3 'ends could prevent uridylation and degradation in plants [71]. But the enzymes responsible for uridylation have not yet been identified. By contrast, as far as the substrate is concerned in animals, the HEN1 methyltransferase only methylated piRNAs, and in some cases, siRNAs [78-80], no miRNAs have been found to be methylated by it. The uridylation has dual effects on the stability of the miRNAs [72] in plants. It seems that the uridylation of the passenger strand equals to addition of a tag to promote degradation, instead the uridylation of the guide strand could serve as a protective modification [72] as 
the uridylated miRNAs favors the Argonaute bindings. However, so far no uniform modification has been yet found in animals. 3' adenylation by the cytoplasmic poly(A) polymerase GLD-2 (also known as TUTase2) could stabilize miRNAs in animals. However, of the animal miRNAs identified to be adenylated, only miR-122 in liver has been characterized for functional consequence [51]. It seems that the GLD-2 non-specifically adds adenosine residues to miRNAs, but only a fraction of these adenylated miRNA species could be stabilized. Similarly, adenylation also occurred in both full length and truncated plant miRNAs [81], but the factors responsible for the adenylation and the related functional consequences are still waiting for elucidation. While diverse nucleotide substitutions, additions and deletions have been detected in animals, and the types and the extents could affect their target recognitions, possible effects on miRNA stability are less predictable [73-75,77].

The third factor is the degradation factor for mature miRNAs. The small RNA degrading nuclease (SDN) is the first exonuclease identified in plants to catalyze 3'- to 5'- cleavage of mature miRNAs [82]. The methylated and uridylated miRNAs are more resistant to degradation by SDN in vitro conditions. By contrast, the 5'- to 3'-exonuclease XRN-2 is responsible for degradation of the single strand and nonincorporated miRNAs in animals [83]. While the functional miRNAs take refuge in RISC complex to avoid degradation, cells apparently have specific mechanisms to release the miRNAs from Argonaute to expose the released miRNAs for degradation. More specifically, the XRN-2mediated degradation is in target availability dependent manner [83]. More recently, it was found that the XRN1 instead of XRN2 could be responsible for miR-382 which contributes to HIV provirus latency and is very unstable in human cells [84]. At normal physiological conditions, the rates of biogenesis and turnover determine the accumulation of functional miRNAs. The appropriate ratio of miRNAs to their targets levels could be maintained to guide RISC reprogramming or turnover. The tight control of the functional miRNA homeostasis is the key to prevent detrimental overexpression or deficiency in miRNAs.

\section{Co-factors enhancing RISC loading or RISC-mediated Silencing}

Although many co-factors were demonstrated to be involved in processing of miRNAs, few co-factors have been identified to be associated with AGO proteins in mammals or in flies to enhance RISC loading or RISC-mediated silencing. The protein components so far identified include putative RNA helicase, RNA binding, heat shock protein, P-body component, endoribonuclease, type I collagen prolyl-4-hydroxylase, and so on. MOV10, a putative RNA helicase, and TNRC6 (-A, - B, -C) are associated with Ago1 and Ago2 and functionally required to mediate miRNA-guided mRNA cleavage $[85,86]$. RBM 4, a putative RNA-binding motif protein can efficiently bind miRNAs and interact with AGO1 and AGO2 in RNA dependent manner, and is required for microRNA-guided gene regulation [87]. Hsp90, a heat shock protein, is associated with AGO2 to efficiently target $\mathrm{hAgO} 2$ to P-bodies and stress granules and thereby enhance microRNA- and siRNA-conferred gene silencing [88]. GW182, a P-body component in Drosophil, and TNGW1, a $210 \mathrm{kDa}$ isoform of the GW182 in human, both interact with AGO1 and the interaction is essential for miRNA-mediated translation repression and mRNA turn over $[89,90]$. The $\alpha-(\mathrm{P} 4 \mathrm{H}-\alpha(\mathrm{I}))$ and $\beta-(\mathrm{P} 4 \mathrm{H}-\beta)$ subunits of type I collagen prolyl-4-hydroxylase (C-P4H(I)) interact with Ago2 and get Ago2 and Ago4 more efficiently hydroxylated than Ago1 and Ago3. The hydroxylation maintains stability of Ago2 and efficiency of RNAi activity [91]. Imp8 functions as a gene silencing factor to stimulate
RISC-mediated silencing by enhancing association of AGO2 complexes to target mRNAs [92]. C3PO, an endoribonuclease, promotes RNAi by facilitating RISC activation in Drosophila [93]. However, it seems that some of them are not essential for RISC loading or target cleavage.

\section{miRNA Intercellular Transportation}

Both siRNAs and miRNAs are trafficking intercellularly instead of being completely restricted in specific cell from which the miRNAs are generated. Recent studies showed that RISC formation and turnover depends on the endosomal pathway [6,94], suggesting a link between RNA silencing and membrane trafficking. Endosome maturation involves a gradual accumulation of internal vesicles which will be finally released into the extracellular milieu called exosomes. The exosome containing the ribonuclease complexes were recently found to carry mRNAs and miRNAs and can transfer [95,96] these nucleic acids into a recipient cell. The purified exosome-like vesicles from the cultured monocytes contained a large number of miRNAs, low levels of AGO2, and high levels of GW182 [94]. P-body component required for miRNAs functions by binding to AGO proteins [96]. Microvesiclesbased transfers of RNAs and proteins from cell to cell are believed to play important roles in intercellular communications. Recent studies have demonstrated intercellular trafficking of miRNAs by a variety of cell types such as normal somatic cells, cancer cells, and stem cells [97101]. The mechanism for miRNA secretion and transfer is just being elucidated. The miRNAs are secreted by a ceramide-dependent secretory pathway and the secreted miRNAs are transferable and functional in the recipient cells [99]. Growing evidences have demonstrated the variety of physiological roles in which the secretory miRNAs may play $[102,103]$, and one of such suggests that the secretory miRNAs could mainly serve in chronic biological events such as the formation of a tumor microenvironment.

\section{MicroRNA and Tumorigenesis}

\section{Global deregulation of miRNAs biogenesis and cancers}

MicroRNAs are predicted to regulate over $50 \%$ of all human proteincoding genes, enabling them function in many physiological and developmental processes [38]. Generally speaking, cancer is partially featured by global down regulation of miRNA expression in accordance with the accumulation of pri- and pre-miRNAs compared to normal tissue [59], while the specific deregulation of certain miRNAs is seen in specific tumor types $[104,105]$. Aberration of any key components in the microRNA pathway as summarized above may contribute to pathology of cancer genesis, progress, and development. Evidences have emerged to link the deregulation of these key components expression or activity and several malignancies, suggesting that the tight control of the microRNA pathway itself at any stages is essential in maintenance of normal metabolism.

\section{Abnormality of key components involved in microRNA pathway and cancer}

Global deregulation of microRNA expression levels has been linked to almost all the type of cancers identified so far, whereas the abnormality of the main protein components in the microRNA pathway accounts for at least one of the main causes for the global deregulation. Copy number abnormalities of some key coding genes such as Dicer, Ago2, Exportin 5 (XPO5), Drosha, TRBP, and DGCR8 that are essential for microRNA biogenesis in human genome have been detected in breast cancer, ovarian cancer, and in melanoma [11,106]. In addition to copy number abnormalities, abnormal expression levels of mature 
form of microRNAs have been observed in some type of cancers due to deregulation of the expression levels of these components involved in the miRNA pathway or their activity alteration. Disruption of miRNAs biogenesis by depletion of any key components in miRNA processing pathway such as Drosha, DGCR8, Dicer, and TRBP, has been linked to promotion of oncogenesis [107-112]. Evidences have arisen in recent years from different animal models or cancer cell lines [107-112]. Under in vitro conditions, mutation of XPO5 inactivation enhances retention of microRNA precursors in the nuclei and accordingly decreases the levels of mature miRNAs in several human tumors [113]. More direct evidences arise from the fact that XPO5 knockdown enhances tumorigenicity of cells injected into mice in accordance with the reverse effect that overexpression of wild type of XPO5 in colorectal cancer cells expressing mutant XPO5 [113]. A serial of other evidences have suggested the essential roles of the normal microRNA biogenesis in inhibition of oncogenesis. In a mouse model of retinoblastoma, monoallelic loss of Dicer1 leads to accumulated tumor formation [108], consistent with the situations in different human tumors in a way that heterozygous instead of homozygous genetic deletion of Dicer 1 frequently occurs, suggesting that DICER1 functions as a haploinsufficient tumor suppressor $[109,110]$. TRBP is one of the essential components in microRNA pathway in ways that maintain the stability of DICER1 and microsatellite, and its concurrent impairment has been indicated in weakness or eradication of miRNA biogenesis and the resulted colon tumors formation/development [112,114,115]. Some small molecule compounds specifically or non-specifically up-regulate expression of miRNAs through direct or indirect interaction with key components involved in miRNA pathway. For example, enoxacin promotes miRNAs biogenesis through enhancing both processing of miRNA precursors to mature form of miRNAs and loading of miRNAs into RISC to increase the efficiency of RNAi [116]. Later on it is found that this compound could significantly inhibit cancer cell growth and metastasis in mouse model [117] due to elevation of a substantial set of miRNAs levels. Recently, a set of iron chelators have been found to interact with Dicer, a key enzyme for the biogenesis of miRNAs with the mechanism that cellular iron chelation enhances the interaction of DICER1 and PolyC binding protein 2 (PCBP2), binding of PCBP2 with miRNA precursors, and processing of precursors into mature miRNAs [118].

\section{Specific deregulation of miRNAs and cancers}

Global deregulation of miRNAs, due to alteration of the key components involved in miRNA pathway, is pretty common in cancers. Another important issue in miRNA mediated cancer is the deregulation of the specific miRNAs.

\section{Specific miRNAs in tumorigenesis and tumor development}

Tumorigenesis initiates from regulation imbalance of cell proliferation and apoptosis in ways that overexpression of oncogenes and/or silencing of tumor suppressor genes. MicroRNAs contribute to regulation of tumorigenesis and tumor development through negatively regulating expression of genes involved in the processes of cell proliferation and apoptosis. At molecular levels, some microRNAs target tumor suppressor genes, while some others are believed to regulate expression of oncogenes. In case of down regulation or null expression of oncogenes-specific miRNAs, the expression of the related target genes will be enhanced, causing or promoting tumorigenesis. Accordingly, overexpression of tumor suppressor genes-specific miRNAs leads to insufficient expression of targets functioning as tumor suppressors, causing tumorigenesis and tumor development. As a general phenomenon, reduced expression levels of miRNAs were detected in tumors relative to that in normal tissues [107]. Although it is not conclusive whether this phenomenon is exactly the cause or the result of the tumorigenesis, based on the previous studies it could suggest that while the majority of miRNAs may be involved in tumor suppression, alteration of miRNAs expression levels might be both the causes and results. As evidence, differentiation state of HL60 cells is maintained partially by enhanced miRNAs expression, and after differentiation treatment, HL60 cells display significantly increased expression of miRNAs [107]. Consistently, activation of the $\mathrm{c}-\mathrm{Myc}$ oncogenic transcription factor (Myc) leads to the widespread repression of miRNA expression due to the fact that Myc directly binds to miRNA promoters, causing tumorigenesis [119], whereas enforced expression of repressed miRNAs diminishes the tumorigenic potential of lymphoma cells. Other study reports that attenuation of miRNAs biogenesis by genetic or RNAi approach promotes tumorigenesis and tumor development in lung cancer model [107]. All together it is demonstrated that extensive reprogramming of the miRNA transcriptome contributes to tumorigenesis.

At physiological levels, some miRNAs function as regulation of angiogenesis, while some miRNAs are thought to be involved in tumor metastasis. In most cases, tumorigenesis originates from deficiency of miRNA generation. Typically, miR-15a and miR-16-1 target Bcl-2, an essential anti-apoptosis factor that tightly regulates proliferation. Deficiency of both miRNA biogenesis leads to overexpression of Bcl-2 and consequently promotes tumorigenesis and tumor development. On the other hand, overexpression of some miRNAs leads to tumorigenesis as well. For examples, miR-221 and miR-222 target KIT protein, protooncogene c-Kit or tyrosine-protein kinase Kit or CD117. Overexpression of both miR-221 and miR-222 dedifferentiates thyrocytes and leads to tumorigenesis [104]. Similarly, miR-150 that targets a transcription factor c-Myb [120] and tumor suppressor EGR2 [52], is dramatically overexpressed in gastric cancer tissues and overexpression of miR150 significantly leads to deficiency of EGR2 expression and therefore promotes gastric cancer cell proliferation [52].

\section{miRNAs and Tumor Metastasis}

Deregulation of some miRNAs leads to tumorigenesis and promotes progression of primary tumors. More importantly, some miRNAs have been linked to tumor metastasis as reviewed already [121-126]. Essentially, abnormal expression of miRNAs including mutation, and down-regulation or overexpression may affect expression levels of their target genes involved in tumor metastasis such as cell migration, invasion, and anoikis resistance in ways that some cancer cell metastasis suppressors are down-regulated due to overexpression of the specific regulatory miRNAs or some metastasis enhancers are overexpressed due to the down regulation of some specific miRNAs. So far a set of miRNAs involved in metastasis have been identified (Table 1).

\section{Metastasis-promoting miRNAs}

Some miRNAs target to suppressors for metastasis named as metastasis-promoting miRNAS, their up-regulation is beneficial to tumor metastasis. miR-221 and miR-222 overexpression induced a down regulation of PTP $\mu$ that is believed to suppress cell migration. Phenotypically, miR-222 and miR-221 induce an increase in cell migration and growth in soft agar in glioma cells. Consistently, the reexpression of PTP $\mu$ gene is able to revert the miR-222 and -221 effects on cell migration. The miR-221/222 overexpession and invasiveness increase is further confirmed in human glioma cancer samples [127], suggesting that the miR-221 and -222 regulate glioma tumorigenesis at least in part through the control of $\mathrm{PTP} \mu$ protein expression. 
Citation: Jiang R, Li Y (2013) Regulation of Mirna Pathway and Roles of Micrornas in Tumorigenesis and Metastasis. Human Genet Embryol S2: 007. doi:10.4172/2161-0436.S2-007

Page 6 of 10

\begin{tabular}{|c|c|c|c|}
\hline miRNA & Role in invasion/metastasis & Cancer type & Target \\
\hline miR-9 & metastasis & colon, melanoma, head, neck & REST, COREST \\
\hline miR-10a & promotion & pancreatic & HOXB1, HOXB4 \\
\hline miR-10b & promotion & $\begin{array}{l}\text { breast, glioblastoma, } \\
\text { esophageal, nasoparyngeal }\end{array}$ & $\begin{array}{l}\text { HOXD10, KLF4, LMP1, BCL2L11/Bim TFAP2C/ } \\
\text { AP-2 } \gamma, \text { CDKN1A/p21, CDKN2A/p16 }\end{array}$ \\
\hline miR-15a & suppression & prostate & BCL-2 \\
\hline miR16 & suppression & prostate & CDK1, CDK2 \\
\hline $\operatorname{miR} 17-92$ & promotion & breast, coloreactal & CTGF, TSP1 \\
\hline miR21 & promotion & $\begin{array}{l}\text { breast, colorectal, gastric, lung, pancreatic, prostate, bladder, } \\
\text { ovarian, hepatocellular }\end{array}$ & $\begin{array}{c}\text { PDCD4, PTEN, CDC25A, RHOB, TIAMI, TPM1, } \\
\text { TPM1, MARCKS, NF1B, SPRY2 }\end{array}$ \\
\hline miR-22 & suppression & breast & CDK6, SIRT1, SP1 \\
\hline miR-25 & promotion & & BIM, BAX and CASPASE-3 \\
\hline miR-27a & promotion & breast & APC, FOXO1 \\
\hline $\mathrm{miR}-31$ & suppression & breast & RHOA, RDX, ITGA5 \\
\hline miR-34a & suppression & gastric & SIRT1-p53 pathway \\
\hline $\begin{array}{l}\text { miR34b and } \\
\text { miR34c }\end{array}$ & metastasis & many different tumor types & TCL1 \\
\hline miR-122 & suppression & hepatocellular & ADAM17, RHOA, RAC1 \\
\hline $\operatorname{miR}-124 a$ & suppression & colon, gastric, haematological & SCP1, PTBP1 \\
\hline $\mathrm{miR}-126$ & suppression & breast, lung & CRK, VEGF \\
\hline miR-129-2 & suppression & colon, endometrical, gastric & $\begin{array}{l}\text { CDK4, SOX4, GALNT1, APC, RAB11, EIF2C3, } \\
\text { CAMTA1 }\end{array}$ \\
\hline miR-137 & suppression & colon, head and neck & LSD1, CDK6, CDC42, MITF \\
\hline miR-141 & promotion & colon, breast, lung, ovary & p38a \\
\hline $\mathrm{miR}-146 \mathrm{a} / \mathrm{b}$ & suppression & breast, pancreatic, glioma, prostate, gastric & EGFR, ROCK1, IRAK1, NFKB1 \\
\hline miR148a & metastasis & colon, melanoma, breast & BCL-2 \\
\hline miR-151 & metastasis & hepatocellular carcinoma & RHOGDIA \\
\hline miR-194 & suppression & liver & CDH2, DNMT3A, HBEGF \\
\hline miR-196b & $\begin{array}{l}\text { dual roles } \\
\text { (suppression/promotion) }\end{array}$ & gastric, leukemia & HOXA9/MEIS1, FAS \\
\hline miR-200c & suppression & colon, breast, lung & ZEB1, ZEB2 \\
\hline miR-205 & suppression & bladder, breast, prostate & $\begin{array}{l}\text { ERBB3, E2F1, E2F5, VEGF-A, IL24, IL32, ZEB1, } \\
\text { ZEB2 }\end{array}$ \\
\hline miR-206 & suppression & breast, rhabdomyosarcoma & ESR1, MET \\
\hline miR-214 & promotion or suppression & melanoma & TFAP2C, MAP2K3, MAPK8 \\
\hline miR-335 & suppression & gastric, breast & BCL2L2, SP1, SOX4, TNC \\
\hline miR-373 & metastasis & breast, testicular germ cell & CD44, LAST2 \\
\hline miR-378 & promotion & breast, glioblastoma & SUFU, FUS-1 \\
\hline $\begin{array}{l}\text { miR-517c/ } \\
\text { miR520g }\end{array}$ & promotion & neuroectodermal brain tumor & WNT upregulation \\
\hline miR-520c & metastasis/invasion & breast & CD44 \\
\hline
\end{tabular}

Table 1: miRNAs involved in tumor invasion and metastasis.

Transcriptionally induced by Twist 1 , a transcription factor, miR-10b has been known for its promotion to breast cancer metastasis and epithelialmesenchymal transition evidenced by the fact that the miR-10b is highly expressed in metastatic breast cancer cells and positively regulates cell migration and invasion in a way that miR-10b inhibits translation of its direct target mRNA encoding for homeobox D10 and in turn activates another well-characterized pro-metastatic gene, RHOC [128]. Some miRNAs target metastasis suppressors, up-regulation of such miRNAs such as miR-182 will be beneficial to tumor metastasis. Metastasis suppressor 1 (MTSS1), one target gene of miR-182, plays an important role in the metastasis of cancers. Evidences from both in vivo and in vitro suggest elevated expression of miR-182 enhances metastasis through the down-regulation of its target gene MTSS1 [129]. Thus, miR-182 and MTSS1 were potential prognostic markers and/or therapeutic targets in HCC. By activation of signal transducer and activator of transcription 3 (STAT3), overexpression of PRL-3 induces expression of miR-21, miR17 and miR-19a. Subsequently, induced expression of these miRNAs is involved in the proliferation and metastasis of colon cancer [130].
MiR-10a functions as enhancement of the colony formation activity, migration and invasion of HeLa and C33A cells through targeting to CHL1. Independent experiment demonstrates that CHL1 serves as a strong repressor for colony formation activity, migration and invasion. Finally, overexpression of CHL1 lacked the 3'UTR abolished the effects of miR-10a, providing an alternative strategy for blocking tumor metastasis [131]. Similarly, induced expression by hypoxia, miR103/107 target the known metastasis suppressors DAPK and KLF4 in CRC cells, resulting in increased cell motility and cell-matrix adhesion and decreased cell-cell adhesion and epithelial marker expression, and suggesting that this regulatory circuit may contribute in part to hypoxia stimulated tumor metastasis. Clinically, a miR-103/107 high, DAPK low, and KLF4 low expression profile has become a featured signature to serve as a prognostic marker for metastasis recurrence and poor survival, strategically suggesting the possibility that disruption of this circuit in blocking the CRC metastasis [132]. MiR-9 targets MMP-14 and thereby downregulate expression of MMP14 and its downstream gene vascular endothelial growth factor (VEGF) in cultured NB cell 
lines SH-SY5Y and SK-N-SH, inhibiting the invasion, metastasis and angiogenesis of NB [133].

\section{Metastasis-inhibiting miRNAs}

Some miRNAs target to metastasis enhancers named as metastasisinhibiting miRNAs accordingly. Down-regulation of these miRNAs initiates or enhances tumor metastasis. MiR-335 suppresses lung and bone metastasis by human cancer cells in vivo through regulation of a set of genes whose collective expression in a large cohort of human tumors is associated with risk of distal metastasis. Thus, miR-335 is identified as metastasis suppressor microRNAs in human breast cancer [134]. MiR-126 suppresses metastatic endothelial recruitment, metastatic angiogenesis and metastatic colonization through coordinate targeting of IGFBP2, PITPNC1 and MERTK- novel pro-angiogenic genes and biomarkers of human metastasis [135]. Human miR-373 and miR-520c have been characterized to stimulate cancer cell migration and invasion in vitro and in vivo mechanistically due to suppression of CD44. miR-373 and miR-520c are implicated as metastasis-promoting miRNAs [20]. MiR-7 is linked to significantly suppress metastasis in highly metastatic GC cell lines and metastatic tissues both in vivo and in vitro through translational inhibition of the insulin-like growth factor-1 receptor (IGF1R) oncogene. Thus, targeting this novel miR7/IGF1R/Snail axis would be helpful as a therapeutic approach to block GC metastasis [136]. Through a Slit-miR-218-Robo1 regulatory circuit, miR-218 functions as suppressor for tumor cell invasion and metastasis in vitro and in vivo. MiR-218 targets Robol, one of several Slit receptors interaction, whereas decrease of miR-218 activates the Slit-Robol pathway through Robol and Slit 2 interaction, triggering tumor metastasis. Thus, targeting miR-218 may provide a strategy for blocking tumor metastasis. [137]. Targeting ILK, miR-625 significantly inhibits invasion and metastasis of gastric cancer cells both in vitro and in vivo [138]. MiR-372 and miR-373, each permits proliferation and tumorigenesis of primary human cells that harbor both oncogenic RAS and active wild-type p53. These miRNAs neutralize p53-mediated CDK inhibition, possibly through directly inhibiting the expression of the tumor-suppressor LATS2. These miRNAs are potential novel oncogenes involved in the tumorigenesis of human testicular germ cells by malfunctioning the p53 pathway, rendering tumorigenic growth continual in the presence of wild-type p53 [139,140].

\section{Perspectives on Regulation of RNAi/miRNA Pathway and Roles of miRNAs in Tumorigenesis and Metastasis}

Significant achievements have been made in understanding regulation of microRNA pathway as well as functions of miRNAs in tumorigenesis and metastasis in recent years, laying down the foundation for further uncovering regulation of gene expression at posttranscriptional level particularly in response to stress and disease initiation such as tumorigenesis and cancer development. Since chemical biology offers a more dynamic way to monitor the activity of specific pathways compared to traditional forward or reverse genetic approach, its emergence to the RNAi/miRNA pathway although still in its infancy, has lead to further understanding on the regulation mechanisms by identification of a number of small molecule modulators targeting to the specific steps in RNAi/miRNA pathway. Further identification of small molecule identifiers and characterization of targets of these modifiers will be essential to further dissect the regulation mechanism. Processing of some specific miRNAs could be regulated in the cell type or physiological condition dependent manners [59,62], while the underlying mechanism of regulation remains to be elucidated. The specific regulation of some miRNAs, particularly those that have been linked to human diseases, is of importance in terms of mechanism of study and development of new therapeutic strategy. Thus, it would be essential to identify small molecules modifiers that could regulate the processing and activity of specific miRNAs. While technically challenged, the success in identification of miR-21-specific inhibitor could shed light on the feasibility of the chemical biology approach in this regard [141]. Besides potential targeting of the protein(s) involved in the regulation of the processing of selective miRNAs, several small molecules could directly bind to RNAs, including miRNAs $[9,119,142]$. Given the diversity of RNA secondary structures among miRNA precursor population, it is plausible to speculate that small molecules targeting to specific pri-, pre- or mature miRNA could be identified. Substantial type of miRNAs has been identified in cancer cells with significant deregulation compared to normal adjacent tissues, caused by transcription factors and chromatin modulators as well as RNA binding proteins and their interacting partners. With development and application of high throughput technologies, more cancer-closelyrelated miRNAs will be identified and further study on metabolic pathways regulated by these cancer-related miRNAs may lead to discovery of unknown pathological mechanisms for tumorigenesis and cancer development. Considering the fact that miRNAs are involved in nearly every cellular process, the identification and characterization of small molecule modulators of RNAi/miRNA pathway will provide novel insights into the fundamentally pathological mechanisms of human diseases particularly cancer. Furthermore, these RNAi modulators, particularly RNAi enhancers, could potentially facilitate the development of RNA interference as a tool for biomedical research and therapeutic interventions.

\section{References}

1. Hannon GJ (2002) RNA interference. Nature 418: 244-251.

2. Bartel DP (2004) MicroRNAs: genomics, biogenesis, mechanism, and function Cell 116: 281-297.

3. Plasterk RH (2006) Micro RNAs in animal development. Cell 124: 877-881.

4. Hwang HW, Wentzel EA, Mendell JT (2007) A hexanucleotide element directs microRNA nuclear import. Science 315: 97-100.

5. Carthew RW, Sontheimer EJ (2009) Origins and Mechanisms of miRNAs and siRNAs. Cell 136: 642-655.

6. Lee YS, Pressman S, Andress AP, Kim K, White JL, et al. (2009) Silencing by small RNAs is linked to endosomal trafficking. Nat Cell Biol 11: 1150-1156.

7. Shi XB, Tepper CG, deVere White RW (2008) Cancerous miRNAs and their regulation. Cell Cycle 7: 1529-1538.

8. Du T, Zamore PD (2005) microPrimer: the biogenesis and function of microRNA Development 132: 4645-4652.

9. Kim VN (2005) MicroRNA biogenesis: coordinated cropping and dicing. Nat Rev Mol Cell Biol 6: 376-385.

10. Kim VN, Han J, Siomi MC (2009) Biogenesis of small RNAs in animals. Nat Rev Mol Cell Biol 10: 126-139.

11. Lee Y, Ahn C, Han J, Choi H, Kim J, et al. (2003) The nuclear RNase III Drosha initiates microRNA processing. Nature 425: 415-419.

12. Berezikov E, Chung WJ, Willis J, Cuppen E, Lai EC (2007) Mammalian mirtron genes. Mol Cell 28: 328-336.

13. Okamura K, Hagen JW, Duan H, Tyler DM, Lai EC (2007) The mirtron pathway generates microRNA-class regulatory RNAs in Drosophila. Cell 130: 89-100.

14. Ruby JG, Jan CH, Bartel DP (2007) Intronic microRNA precursors that bypass Drosha processing. Nature 448: 83-86.

15. Babiarz JE, Ruby JG, Wang Y, Bartel DP, Blelloch R (2008) Mouse ES cells express endogenous shRNAs, siRNAs, and other Microprocessor-independent, Dicer-dependent small RNAs. Genes Dev 22: 2773-2785. 
Citation: Jiang R, Li Y (2013) Regulation of Mirna Pathway and Roles of Micrornas in Tumorigenesis and Metastasis. Human Genet Embryol S2: 007. doi:10.4172/2161-0436.S2-007

16. Ender C, Krek A, Friedländer MR, Beitzinger M, Weinmann L et al. (2008) A human snoRNA with microRNA-like functions. Mol Cell 32: 519-528.

17. Saraiya AA, Wang CC (2008) snoRNA, a novel precursor of microRNA in Giardia lamblia. PLoS Pathog 4: e1000224.

18. Cole C, Sobala A, Lu C, Thatcher SR, Bowman A, et al. (2009) Filtering of deep sequencing data reveals the existence of abundant Dicer-dependent smal RNAs derived from tRNAs. RNA 15: 2147-2160.

19. Miyoshi K, Miyoshi T, Siomi H (2010) Many ways to generate microRNA-like small RNAs: non-canonical pathways for microRNA production. Mol Genet Genomics 284: 95-103.

20. Okada C, Yamashita E, Lee SJ, Shibata S, Katahira J et al. (2009) A highresolution structure of the pre-microRNA nuclear export machinery. Science 326: $1275-1279$

21. Bohnsack MT, Czaplinski K, Gorlich D (2004) Exportin 5 is a RanGTPdependent dsRNA-binding protein that mediates nuclear export of pre-miRNAs. RNA 10: 185-191.

22. Maniataki E, Mourelatos Z (2005) A human, ATP-independent, RISC assembly machine fueled by pre-miRNA. Genes Dev 19: 2979-2990.

23. Lee Y, Hur I, Park SY, Kim YK, Suh MR, et al. (2006) The role of PACT in the RNA silencing pathway. EMBO J 25: 522-532.

24. Matranga C, Tomari Y, Shin C, Bartel DP, Zamore PD (2005) Passenger-strand cleavage facilitates assembly of siRNA into Ago2-containing RNAi enzyme complexes. Cell 123: 607-620.

25. Czech B, Zhou R, Erlich Y, Brennecke J, Binari R, et al. (2009) Hierarchical rules for Argonaute loading in Drosophila. Mol Cell 36: 445-456.

26. Guo L, Liu Y, Bai Y, Sun Y, Xiao F, et al. (2010) Gene expression profiling of drug-resistant small cell lung cancer cells by combining microRNA and cDNA expression analysis. Eur J Cancer 46: 1692-1702.

27. Okamura K, Phillips MD, Tyler DM, Duan H, Chou YT, et al. (2008). The regulatory activity of microRNA* species has substantial influence on microRNA and 3' UTR evolution. Nat Struct Mol Biol 15: 354-363.

28. Seitz H, Ghildiyal M, Zamore PD (2008) Argonaute loading improves the 5 precision of both MicroRNAs and their miRNA* strands in flies. Curr Biol 18 147-151.

29. Kawahara $Y$, Zinshteyn B, Sethupathy $P$, lizasa H, Hatzigeorgiou AG, et al (2007) Redirection of silencing targets by adenosine-to-inosine editing of miRNAs. Science 315 : 1137-1140.

30. Schwarz DS, Hutvágner G, Du T, Xu Z, Aronin N, et al. (2003) Asymmetry in the assembly of the RNAi enzyme complex. Cell 115: 199-208.

31. Cao X, Yeo G, Muotri AR, Kuwabara T, Gage FH (2006) Noncoding RNAs in the mammalian central nervous system. Annu Rev Neurosci 29: 77-103.

32. Sevignani C, Calin GA, Siracusa LD, Croce CM (2006) Mammalian microRNAs: a small world for fine-tuning gene expression. Mamm Genome 17: 189-202.

33. He L, Hannon GJ (2004) MicroRNAs: small RNAs with a big role in gene regulation. Nat Rev Genet 5: 522-531.

34. Duan R, Pak C, Jin P (2007) Single nucleotide polymorphism associated with mature miR-125a alters the processing of pri-miRNA. Hum Mol Genet 16: 11241131.

35. Jazdzewski K, Murray EL, Franssila K, Jarzab B, Schoenberg DR, et al (2008) Common SNP in pre-miR-146a decreases mature miR expression and predisposes to papillary thyroid carcinoma. Proc Natl Acad Sci U S A 105 7269-7274.

36. Sun G, Yan J, Noltner K, Feng J, Li H, et al. (2009) SNPs in human miRNA genes affect biogenesis and function. RNA 15: 1640-1651.

37. Hu Z, Chen J, Tian T, Zhou X, Gu H, et al. (2008) Genetic variants of miRNA sequences and non-small cell lung cancer survival. J Clin Invest 118: 26002608.

38. Saito Y, Liang G, Egger G, Friedman JM, Chuang JC, et al. (2006) Specific activation of microRNA-127 with downregulation of the proto-oncogene BCL6 by chromatin-modifying drugs in human cancer cells. Cancer Cell 9: 435-443.

39. Maas S, Rich A, Nishikura K (2003) A-to-I RNA editing: recent news and residual mysteries. J Biol Chem 278: 1391-1394.
40. Desterro JM, Keegan LP, Lafarga M, Berciano MT, O'Connell M, et al. (2003) Dynamic association of RNA-editing enzymes with the nucleolus. J Cell Sci 116: $1805-1818$

41. Luciano DJ, Mirsky H, Vendetti NJ, Maas S (2004) RNA editing of a miRNA precursor. RNA 10: 1174-1177.

42. Yang W, Chendrimada TP, Wang Q, Higuchi M, Seeburg PH, et al. (2006) Modulation of microRNA processing and expression through RNA editing by ADAR deaminases. Nat Struct Mol Biol 13: 13-21.

43. Scadden AD (2005) The RISC subunit Tudor-SN binds to hyper-edited doublestranded RNA and promotes its cleavage. Nat Struct Mol Biol 12: 489-496.

44. Fukuda T, Yamagata K, Fujiyama S, Matsumoto T, Koshida I, et al. (2007) DEAD-box RNA helicase subunits of the Drosha complex are required fo processing of rRNA and a subset of microRNAs. Nat Cell Biol 9: 604-611.

45. Davis BN, Hilyard AC, Lagna G, Hata A (2008) SMAD proteins control DROSHA-mediated microRNA maturation. Nature 454: 56-61.

46. Sabin LR, Zhou R, Gruber JJ, Lukinova N, Bambina S, et al. (2009) Ars2 regulates both miRNA- and siRNA- dependent silencing and suppresses RNA virus infection in Drosophila. Cell 138: 340-351.

47. Sakamoto S, Aoki K, Higuchi T, Todaka H, Morisawa K, et al. (2009) The NF90NF45 complex functions as a negative regulator in the microRNA processing pathway. Mol Cell Biol 29: 3754-3769.

48. Suzuki HI, Yamagata K, Sugimoto K, Iwamoto T, Kato S, et al. (2009) Modulation of microRNA processing by p53. Nature 460: 529-533.

49. Yamagata K, Fujiyama S, Ito S, Ueda T, Murata T, et al. (2009) Maturation of microRNA is hormonally regulated by a nuclear receptor. Mol Cell 36: 340-347.

50. Wu H, Sun S, Tu K, Gao Y, Xie B, et al. (2010) A splicing-independent function of SF2/ASF in microRNA processing. Mol Cell 38: 67-77.

51. Katoh T, Sakaguchi Y, Miyauchi K, Suzuki T, Kashiwabara S, et al. (2009) Selective stabilization of mammalian microRNAs by 3' adenylation mediated by the cytoplasmic poly(A) polymerase GLD-2. Genes Dev 23: 433-438.

52. Wu Q, Jin H, Yang Z, Luo G, Lu Y, et al. (2010) MiR-150 promotes gastric cancer proliferation by negatively regulating the pro-apoptotic gene EGR2. Biochem Biophys Res Commun 392: 340-345.

53. Paroo Z, Pertsemlidis A (2009) microRNAs mature with help from cance biology. Genome Biol 10: 310.

54. Huang J, Wang HB, Yao L, Zheng XM, Radmark O (2008) Dicer interacts with Wig-1 protein. Fen Zi Xi Bao Sheng Wu Xue Bao 41: 376-382.

55. Diederichs S, Haber DA (2007) Dual role for argonautes in microRNA processing and posttranscriptional regulation of microRNA expression. Cell 131: $1097-1108$

56. Cheloufi S, Dos Santos CO, Chong MM, Hannon GJ (2010)A dicer-independent miRNA biogenesis pathway that requires Ago catalysis. Nature 465: 584-589.

57. Cifuentes D, Xue H, Taylor DW, Patnode H, Mishima Y, et al. (2010) A nove miRNA processing pathway independent of Dicer requires Argonaute2 catalytic activity. Science 328: 1694-1698.

58. Ruggiero T, Trabucchi M, De Santa F, Zupo S, Harfe BD, et al. (2009) LPS induces $\mathrm{KH}$-type splicing regulatory protein-dependent processing of microRNA-155 precursors in macrophages. Faseb J 23: 2898-2908.

59. Newman MA, Thomson JM, Hammond SM (2008) Lin-28 interaction with the Let-7 precursor loop mediates regulated microRNA processing. RNA 14: 1539 1549 .

60. Piskounova E, Viswanathan SR, Janas M, LaPierre RJ, Daley GQ, et al. (2008) Determinants of microRNA processing inhibition by the developmentally regulated RNA-binding protein Lin28. J Biol Chem 283: 21310-21314.

61. Rybak A, Fuchs H, Smirnova L, Brandt C, Pohl EE, et al. (2008) A feedback loop comprising lin-28 and let-7 controls pre-let-7 maturation during neura stem-cell commitment. Nat Cell Biol 10: 987-993.

62. Viswanathan SR, Daley GQ, Gregory RI (2008) Selective blockade of microRNA processing by Lin28. Science 320 : $97-100$.

63. Heo I, Joo C, Kim YK, Ha M, Yoon MJ, et al. (2009) TUT4 in concert with Lin28 suppresses microRNA biogenesis through pre-microRNA uridylation. Cell 138 696-708. 
Citation: Jiang R, Li Y (2013) Regulation of Mirna Pathway and Roles of Micrornas in Tumorigenesis and Metastasis. Human Genet Embryol S2: 007. doi:10.4172/2161-0436.S2-007

Page 9 of 10

64. Hagan JP, Piskounova E, Gregory RI (2009) Lin28 recruits the TUTase Zcchc11 to inhibit let-7 maturation in mouse embryonic stem cells. Nat Struct Mol Biol 16: $1021-1025$

65. Lehrbach NJ, Armisen J, Lightfoot HL, Murfitt KJ, Bugaut A, et al. (2009) LIN28 and the poly $(U)$ polymerase PUP-2 regulate let-7 microRNA processing in Caenorhabditis elegans. Nat Struct Mol Biol 16: 1016-1020.

66. Guil S, Cáceres JF (2007) The multifunctional RNA-binding protein hnRNP A1 is required for processing of miR-18a. Nat Struct Mol Biol 14: 591-596.

67. Michlewski G, Guil S, Semple CA, Cáceres JF (2008) Posttranscriptional regulation of miRNAs harboring conserved terminal loops. Mol Cell 32: 383 393.

68. Grishok A, Pasquinelli AE, Conte D, Li N, Parrish S, et al. (2001) Genes and mechanisms related to RNA interference regulate expression of the smal temporal RNAs that control C. elegans developmental timing. Cell 106: 23-34.

69. O'Carroll D, Mecklenbrauker I, Das PP, Santana A, Koenig U, et al. (2007) A Slicer-independent role for Argonaute 2 in hematopoiesis and the microRNA pathway. Genes Dev 21: 1999-2004.

70. Vaucheret $H$, Vazquez F, Crété $P$, Bartel DP (2004) The action of ARGONAUTE1 in the miRNA pathway and its regulation by the miRNA pathway are crucial for plant development. Genes Dev 18: 1187-1197.

71. Yu B, Yang Z, Li J, Minakhina S, Yang M, et al. (2005) Methylation as a crucia step in plant microRNA biogenesis. Science 307: 932-935.

72. Li J, Yang Z, Yu B, Liu J, Chen X (2005) Methylation protects miRNAs and siRNAs from a 3'-end uridylation activity in Arabidopsis. Curr Biol 15: 1501 1507.

73. Landgraf P, Rusu M, Sheridan R, Sewer A, lovino N, et al. (2007) A mammalian microRNA expression atlas based on small RNA library sequencing. Cell 129: 1401-1414.

74. Reid JG, Nagaraja AK, Lynn FC, Drabek RB, Muzny DM, et al. (2008) Mouse let-7 miRNA populations exhibit RNA editing that is constrained in the 5'-seed/ cleavage/anchor regions and stabilize predicted mmu-let-7a:mRNA duplexes. Genome Res 18: 1571-1581.

75. Ruby JG, Jan C, Player C, Axtell MJ, Lee W, et al. (2006) Large-scale sequencing reveals 21U-RNAs and additional microRNAs and endogenous siRNAs in C. elegans. Cell 127: 1193-1207.

76. Azuma-Mukai A, Oguri H, Mituyama T, Qian ZR, Asai K, et al. (2008) Characterization of endogenous human Argonautes and their miRNA partners in RNA silencing. Proc Natl Acad Sci U S A 105: 7964-7969.

77. Morin RD, O'Connor MD, Griffith M, Kuchenbauer F, Delaney A, et al. (2008) Application of massively parallel sequencing to microRNA profiling and discovery in human embryonic stem cells. Genome Res 18: 610-621.

78. Horwich MD, Li C, Matranga C, Vagin V, Farley G, et al. (2007) The Drosophila RNA methyltransferase, DmHen1, modifies germline piRNAs and singlestranded siRNAs in RISC. Curr Biol 17: 1265-1272.

79. Kirino Y, Mourelatos $Z$ (2007) The mouse homolog of HEN1 is a potential methylase for Piwi-interacting RNAs. RNA 13: 1397-1401.

80. Saito K, Sakaguchi Y, Suzuki T, Suzuki T, Siomi H, et al. (2007) Pimet, the Drosophila homolog of HEN1, mediates 2'-O-methylation of Piwi- interacting RNAs at their 3' ends. Genes Dev 21: 1603-1608.

81. Lu S, Sun YH, Chiang VL (2009) Adenylation of plant miRNAs. Nucleic Acids Res 37: 1878-1885.

82. Ramachandran V, Chen X (2008) Degradation of microRNAs by a family of exoribonucleases in Arabidopsis. Science 321: 1490-1492.

83. Chatterjee S, Grosshans H (2009) Active turnover modulates mature microRNA activity in Caenorhabditis elegans. Nature 461: 546-549.

84. Bail S, Swerdel M, Liu H, Jiao X, Goff LA, et al. (2010) Differential regulation of microRNA stability. RNA 16: 1032-1039.

85. Meister G, Landthaler M, Peters L, Chen PY, Urlaub H, et al. (2005) Identification of novel argonaute-associated proteins. Curr Biol 15: 2149-2155.

86. Landthaler M, Gaidatzis D, Rothballer A, Chen PY, Soll SJ, et al. (2008) Molecular characterization of human Argonaute-containing ribonucleoprotein complexes and their bound target mRNAs. RNA 14: 2580-2596.

87. Höck J, Weinmann L, Ender C, Rüdel S, Kremmer E, et al. (2007) Proteomic and functional analysis of Argonaute-containing mRNA-protein complexes in human cells. EMBO Rep 8: 1052-1060.

88. Pare JM, Tahbaz N, López-Orozco J, LaPointe P, Lasko P, et al. (2009) Hsp90 regulates the function of argonaute 2 and its recruitment to stress granules and P-bodies. Mol Biol Cell 20: 3273-3284.

89. Eulalio A, Behm-Ansmant I, Izaurralde E (2007) P bodies: at the crossroads of post-transcriptional pathways. Nat Rev Mol Cell Biol 8: 9-22.

90. Li S, Lian SL, Moser JJ, Fritzler ML, Fritzler MJ, et al. (2008) Identification of GW182 and its novel isoform TNGW1 as translational repressors in Ago2mediated silencing. J Cell Sci 121: 4134-4144.

91. Qi HH, Ongusaha PP, Myllyharju J, Cheng D, Pakkanen O, et al. (2008) Prolyl 4-hydroxylation regulates Argonaute 2 stability. Nature 455: 421-424.

92. Weinmann L, Höck J, Ivacevic T, Ohrt T, Mütze J, et al. (2009) Importin 8 is a gene silencing factor that targets argonaute proteins to distinct mRNAs. Cell 136: 496-507.

93. Liu Y, Ye X, Jiang F, Liang C, Chen D, et al. (2009) C3PO, an endoribonuclease that promotes RNAi by facilitating RISC activation. Science 325: 750-753.

94. Gibbings DJ, Ciaudo C, Erhardt M, Voinnet O (2009) Multivesicular bodies associate with components of miRNA effector complexes and modulate miRNA activity. Nat Cell Biol 11: 1143-1149.

95. Stoorvogel W, Kleijmeer MJ, Geuze HJ, Raposo G (2002) The biogenesis and functions of exosomes. Traffic 3: 321-330.

96. Skog J, Würdinger T, van Rijn S, Meijer DH, Gainche L, et al. (2008) Glioblastoma microvesicles transport RNA and proteins that promote tumour growth and provide diagnostic biomarkers. Nat Cell Biol 10: 1470-1476.

97. Yuan A, Farber EL, Rapoport AL, Tejada D, Deniskin R, et al. (2009) Transfer of microRNAs by embryonic stem cell microvesicles. PLoS One 4: e4722.

98. Kren BT, Wong PY, Shiota A, Zhang X, Zeng Y, et al. (2009) Polysome trafficking of transcripts and microRNAs in regenerating liver after partial hepatectomy Am J Physiol Gastrointest Liver Physiol 297: 1181-1192.

99. Kosaka N, Iguchi H, Yoshioka Y, Takeshita F, Matsuki Y, et al. (2010) Secretory mechanisms and intercellular transfer of microRNAs in living cells. J Biol Chem 285: $17442-17452$

100.Pegtel DM, Cosmopoulos K, Thorley-Lawson DA, van Eijndhoven MA Hopmans ES, et al. (2010) Functional delivery of viral miRNAs via exosomes. Proc Natl Acad Sci U S A 107: 6328-6333.

101. Yue J, Tigyi G (2006) MicroRNA trafficking and human cancer. Cancer Bio Ther 5: 573-578.

102.Zernecke A, Bidzhekov K, Noels H, Shagdarsuren E, Gan L, et al. (2009) Delivery of microRNA-126 by apoptotic bodies induces CXCL 12-dependent vascular protection. Sci Signal 2: ra81.

103. Rechavi O, Erlich Y, Amram H, Flomenblit L, Karginov FV, et al. (2009) Cel contact-dependent acquisition of cellular and viral nonautonomously encoded small RNAs. Genes Dev 23: 1971-1979.

104. He H, Jazdzewski K, Li W, Liyanarachchi S, Nagy R, et al. (2005) The role of microRNA genes in papillary thyroid carcinoma. Proc Natl Acad Sci U S A 102 19075-19080.

105. Calin GA, Ferracin M, Cimmino A, Di Leva G, Shimizu M, et al. (2005) A MicroRNA signature associated with prognosis and progression in chronic lymphocytic leukemia. N Engl J Med 353: 1793-1801.

106. Trabucchi M, Briata P, Garcia-Mayoral M, Haase AD, Filipowicz W, et al. (2009) The RNA-binding protein KSRP promotes the biogenesis of a subset of microRNAs. Nature 459: 1010-1014.

107. Kumar MS, Lu J, Mercer KL, Golub TR, Jacks T (2007) Impaired microRNA processing enhances cellular transformation and tumorigenesis. Nat Genet 39: 673-677.

108. Lambertz I, Nittner D, Mestdagh P, Denecker G, Vandesompele J, et al. (2010) Monoallelic but not biallelic loss of Dicer1 promotes tumorigenesis in vivo. Cell Death Differ 17: 633-641.

109. Hill DA, Ivanovich J, Priest JR, Gurnett CA, Dehner LP, et al. (2009) DICER1 mutations in familial pleuropulmonary blastoma. Science 325: 965

110. Kumar MS, Pester RE, Chen CY, Lane K, Chin C, et al. (2009) Dicer1 functions as a haploinsufficient tumor suppressor. Genes Dev 23: 2700-2704. 
Citation: Jiang R, Li Y (2013) Regulation of Mirna Pathway and Roles of Micrornas in Tumorigenesis and Metastasis. Human Genet Embryol S2: 007. doi:10.4172/2161-0436.S2-007

Page 10 of 10

111. Martello G, Rosato A, Ferrari F, Manfrin A, Cordenonsi M, et al. (2010) A MicroRNA targeting dicer for metastasis control. Cell 141: 1195-1207.

112. Melo SA, Ropero S, Moutinho C, Aaltonen LA, Yamamoto H, et al. (2009) A TARBP2 mutation in human cancer impairs microRNA processing and DICER1 function. Nat Genet 41: 365-370.

113. Melo SA, Moutinho C, Ropero S, Calin GA, Rossi S, et al. (2010) A genetic defect in exportin- 5 traps precursor microRNAs in the nucleus of cancer cells. Cancer Cell 18: 303-315.

114. Chendrimada TP, Gregory RI, Kumaraswamy E, Norman J, Cooch N, et al. (2005) TRBP recruits the Dicer complex to Ago2 for microRNA processing and gene silencing. Nature 436: 740-744

115. Garre P, Pérez-Segura P, Díaz-Rubio E, Caldés T, de la Hoya M (2010) Reassessing the TARBP2 mutation rate in hereditary nonpolyposis colorectal cancer. Nat Genet 42: 817-818.

116. Shan G, Li Y, Zhang J, Li W, Szulwach KE, et al. (2008) A small molecule enhances RNA interference and promotes microRNA processing. Nat Biotechnol 26: 933-940.

117. Melo S, Villanueva A, Moutinho C, Davalos V, Spizzo R, et al. (2011) Small molecule enoxacin is a cancer-specific growth inhibitor that acts by enhancing TAR RNA-binding protein 2-mediated microRNA processing. Proc Natl Acad Sci U S A 108: 4394-4399.

118. Li Y, Lin L, Li Z, Ye X, Xiong K, et al. (2012) Iron homeostasis regulates the activity of the microRNA pathway through poly $(\mathrm{C})$-binding protein 2 . Cell Metab 15: 895-904.

119. Chang TC, Yu D, Lee YS, Wentzel EA, Arking DE, et al. (2008) Widespread microRNA repression by Myc contributes to tumorigenesis. Nat Genet 40: 43 50 .

120.Xiao C, Calado DP, Galler G, Thai TH, Patterson HC, et al. (2007) MiR-150 controls $\mathrm{B}$ cell differentiation by targeting the transcription factor $\mathrm{c}-\mathrm{Myb}$. Cell 131: $146-159$

121. Ma L, Weinberg RA (2008) MicroRNAs in malignant progression. Cell Cycle 7: $570-572$.

122. Dumont N, Tlsty TD (2009) Reflections on miR-ing effects in metastasis Cancer Cell 16: 3-4.

123. Nicoloso MS, Spizzo R, Shimizu M, Rossi S, Calin GA (2009) MicroRNAsthe micro steering wheel of tumour metastases. Nat Rev Cancer 9: 293-302.

124. Baranwal S, Alahari SK (2010) miRNA control of tumor cell invasion and metastasis. Int J Cancer 126: 1283-1290.

125. Santarpia L, Nicoloso M, Calin GA (2010) MicroRNAs: a complex regulatory network drives the acquisition of malignant cell phenotype. Endocr Relat Cancer 17: F51-75.

126. Garofalo M, Quintavalle C, Romano G, Croce CM, Condorelli G (2012) miR221/222 in cancer: their role in tumor progression and response to therapy. Curr Mol Med 12: 27-33.

127. Quintavalle C, Garofalo M, Zanca C, Romano G, laboni M, et al. (2012) miR-
221/222 overexpession in human glioblastoma increases invasiveness by targeting the protein phosphate PTP $\mu$. Oncogene 31: 858-868.

128. Ma L, Teruya-Feldstein J, Weinberg RA (2007) Tumour invasion and metastasis initiated by microRNA-10b in breast cancer. Nature 449: 682-688.

129.Wang J, Li J, Shen J, Wang C, Yang L, et al. (2012) MicroRNA-182 downregulates metastasis suppressor 1 and contributes to metastasis of hepatocellular carcinoma. BMC Cancer 12: 227

130. Zhang J, Xiao Z, Lai D, Sun J, He C, et al. (2012) miR-21, miR-17 and miR-19a induced by phosphatase of regenerating liver-3 promote the proliferation and metastasis of colon cancer. $\mathrm{Br} \mathrm{J}$ Cancer 107: 352-359.

131. Long MJ, Wu FX, Li P, Liu M, Li X, et al. (2012) MicroRNA-10a targets CHL1 and promotes cell growth, migration and invasion in human cervical cance cells. Cancer Lett 324: 186-196.

132. Chen HY, Lin YM, Chung HC, Lang YD, Lin CJ, et al. (2012) miR-103/107 promote metastasis of colorectal cancer by targeting the metastasis suppressors DAPK and KLF4. Cancer Res 72: 3631-3641.

133.Zhang H, Qi M, Li S, Qi T, Mei H, et al. (2012) microRNA-9 targets matrix metalloproteinase 14 to inhibit invasion, metastasis, and angiogenesis of neuroblastoma cells. Mol Cancer Ther 11: 1454-1466.

134. Tavazoie SF, Alarcón C, Oskarsson T, Padua D, Wang Q, et al. (2008) Endogenous human microRNAs that suppress breast cancer metastasis. Nature 451: 147-152.

135.Png KJ, Halberg N, Yoshida M, Tavazoie SF (2011) A microRNA regulon that mediates endothelial recruitment and metastasis by cancer cells. Nature 481 : 190-194.

136.Zhao X, Dou W, He L, Liang S, Tie J, et al. (2012) MicroRNA-7 functions as an anti-metastatic microRNA in gastric cancer by targeting insulin-like growth factor-1 receptor. Oncogene.

137. Tie J, Pan Y, Zhao L, Wu K, Liu J, et al. (2010) MiR-218 inhibits invasion and metastasis of gastric cancer by targeting the Robo1 receptor. PLoS Genet 6 : e1000879.

138. Wang M, Li C, Nie H, Lv X, Qu Y, et al. (2012) Down-regulated miR-625 suppresses invasion and metastasis of gastric cancer by targeting ILK. FEBS Lett 586: 2382-2388.

139. Voorhoeve PM, le Sage C, Schrier M, Gillis AJ, Stoop H et al. (2006) Genetic screen implicates miRNA-372 and miRNA-373 as oncogenes in testicula germ cell tumors. Cell. 124: 1169-1181.

140. Voorhoeve PM, le Sage C, Schrier M, Gillis AJ, Stoop H, et al. (2007) A genetic screen implicates miRNA-372 and miRNA-373 as oncogenes in testicula germ cell tumors. Adv Exp Med Biol 604: 17-46.

141. Huang Q, Gumireddy K, Schrier M, le Sage C, Nagel R, et al. (2008) The microRNAs miR-373 and miR-520c promote tumour invasion and metastasis. Nat Cell Biol 10: 202-210.

142. Calin GA, Sevignani C, Dumitru CD, Hyslop T, Noch E, et al. (2004) Human microRNA genes are frequently located at fragile sites and genomic regions involved in cancers. Proc Natl Acad Sci U S A 101: 2999-3004.

\footnotetext{
This article was originally published in a special issue, Epigenetics, Stem Cells and Tumorigenicity handled by Editor(s). Dr. Yue Zhang, Harvard Medical School, USA; Yujing Li, Emory University School of Medicine, USA; Yanhong Ji, Xian Jiaotong University, China
} 